\title{
Optimal Space-Time Transceiver Design for Selective Wireless Broadcast With Channel State Information
}

\author{
Jianzhong Zhang, Member, IEEE, Akbar M. Sayeed, Senior Member, IEEE, and Barry D. Van Veen, Fellow, IEEE
}

\begin{abstract}
Selective broadcast schemes for point-to-multiple point transmission of identical information to several selected users are studied for a code-division multiple-access wireless system. The channel states for all selected users are assumed known at both the transmitter and the receivers. The goal is to minimize total transmit power while satisfying minimum received signal-to-noise ratio (SNR) requirements. Three designs, namely, time-only, space-time and space-only, are investigated. In the time-only design no spatial diversity is available, and we solve the optimal transmit signature code by developing iterative least distance programming (ILDP) and linear programming (LP) algorithms. In the space-time design, transmit antennas are exploited in addition to the temporal dimension, and we show the ILDP algorithm is still applicable. The LP algorithm can also be adapted with the integration of space-time block codes, which we term the space-time block coding LP (STC-LP) algorithm. In the space-only design, only the spatial dimension is available and we study the optimization of the transmit antenna weights to satisfy the users' SNR requirements. We show that the STC-LP algorithm applies in this case. We also propose an iterative spatial diagonalization algorithm to explore the unique structure of the space-only problem.
\end{abstract}

Index Terms-Broadcast, code-division multiple access (CDMA), multicast, space-time block codes, space-time processing, transmit-receive joint optimization.

\section{NOMENCLATURE}

c Transmit signature code.

$\mathbf{r}_{k} \quad$ Receive filter for $k$ th user.

$\eta_{k} \quad$ Required signal-to-noise ratio at $k$ th user.

$(.)^{H} \quad$ Hermitian transpose operator for vectors and matrices.

$(.)^{*} \quad$ Complex conjugate operator.

$K \quad$ Number of mobile stations in the cell.

$L \quad$ Length of the maximum multipath delay in chips.

$N \quad$ Number of antennas at the transmitter.

$P \quad$ Signature code length, or spreading gain.

\section{INTRODUCTION}

$\mathbf{T}$ HE field of wireless communication has been dominated by study of transmission of unicast information. The term unicast means that information is intended for only one receiver. Most voice and data traffic in cellular systems falls into this

Manuscript received January 3, 2002; revised April 28, 2003; accepted October 25, 2003. The editor coordinating the review of this paper and approving it for publication is Y.-D. Yao This paper was presented in part at the 2002 ICASSP conference. This work was supported in part by National Science Foundation under Grant ECS-9979448.

J. Zhang is with the Nokia Research Center, Irving, TX 75039 USA (e-mail: Charlie.Zhang@nokia.com).

A. M. Sayeed and B. Van Veen are with the Department of Electrical and Computer Engineering, University of Wisconsin at Madison, Madison, WI 53706 USA.

Digital Object Identifier 10.1109/TWC.2004.837452

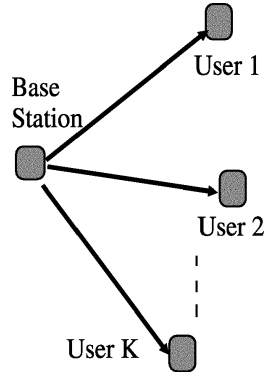

Fig. 1. Base station transmitting identical information to $K$ users.

category. However, there are situations that require selective broadcast, that is, the same information is intended for multiple selected receivers. Examples include a base station sending weather updates, road information to several selected users in a cell, or a multiuser gaming server updating the game status to all the users in a cell [1]. Other types of selective broadcast include the control channel of a cellular system, HDTV broadcasting [2], and multicasting in ad hoc military wireless networks [3].

In order to contrast broadcast and unicast schemes, consider the scenario depicted in Fig. 1, where the base station is sending the same information to all users. We assume that all transmit and receive antennas are omnidirectional. One signaling scheme is to transmit this identical information to individual users as if it was unicast information, via multiplexing methods such as time-, frequency-, or code-division multiple access (CDMA). In this case, the same information is transmitted in different signal space dimensions associated with different users. Contrast this unicast approach with the simple broadcast approach in which the same signal space dimension is used to send the identical information to all the users. Suppose $P_{k}$ is the transmit power needed to provide the $k$ th user with the required signal-to-noise ratio (SNR). If a multiplexed unicast scheme is adopted, the total power required at the transmitter is $P_{\text {total }}=\sum_{k} P_{k}$, whereas simple broadcasting drops the total power to $P_{\text {total }}=\max _{k}\left\{P_{k}\right\}$ [3]. Furthermore, broadcasting uses fewer spectral resources than unicast since the same signal space dimension is used to send the identical information to all the users.

In this paper we investigate the optimization of transceiver designs for selective broadcast in a CDMA wireless system. The optimization is performed jointly on the transmit signature code and receive filters. We assume perfect knowledge of the channel states of all the selected users at the transmitter and receivers. In a time-division duplex (TDD) system, since the channel is the same for the uplink and downlink, the downlink channel states can be estimated at the transmitter if the duplexing time is small compared with the coherence time of the channel [4]. In 
a frequency-division duplex (FDD) system, on the other hand, a feedback mechanism can be introduced to bring the channel state information from receivers to the transmitter [4], [5]. Note that in all cases we assume that the receiver has a single antenna while the transmitter may have multiple antennas.

We consider three categories of transceiver designs: timeonly, space-time, and space-only designs. In the time-only design, a single antenna is assumed at the transmitter. We solve for the optimal transmit signature code and receive filters to satisfy the SNR requirement for all selected users. Two algorithms are developed for this problem. The first approach is named the iterative least distance programming (ILDP) algorithm and solves for the transmit signature code and receive filters iteratively. The second approach is termed the linear programming (LP) algorithm and reduces the original optimization problem to an approximate LP problem via simultaneous diagonalization of channel matrices. The LP algorithm is more computationally efficient than the ILDP algorithm and offers approximately the same performance.

In the space-time design, we add multiple transmit antennas to exploit the spatial dimension. This problem is of interest since cellular systems often have multiple antennas at the base station. We show that the ILDP algorithm developed for the time-only design can be easily extended to the space-time design. On the other hand, since the channels of different users do not share a common eigen structure in the spatial dimension, the computationally efficient LP algorithm is not directly applicable in the space-time design. Nevertheless, the LP algorithm can be adapted for the space-time design with the integration of the space-time block codes described in [6] and [7], which we term space-time block code-based linear programming (STC-LP). Compared with the computationally more expensive ILDP algorithm, STC-LP suffers a slight performance loss.

In the space-only design, we address the problem of designing optimal transmit antenna weights, assuming a fixed signature code at the transmitter and fixed filters at the receivers. We show that the STC-LP algorithm can be adapted to this problem, but the ILDP algorithm is not applicable. We also propose an iterative spatial decomposition (ISD) algorithm to exploit the unique structure of this problem.

We employ chip-rate sampled channel representation for slow fading wireless CDMA channels. Channel state information is assumed to be known at both transmitter and receiver. We also assume that the signature code length $P$ is much longer than the multipath delay length $L$, i.e., $P \gg L$, so that intersymbol interference in the system is negligible. Binary phase-shift keying modulation is adopted throughout this paper. The thermal noise is assumed to be complex white Gaussian with variance $\sigma^{2}$.

\section{TIME-ONLY DESIGN}

Consider the simple broadcast system shown in Fig. 1. The base station broadcasts information to $K$ mobile users in the cell simultaneously. Let $\mathbf{r}_{1}, \mathbf{r}_{2}, \ldots, \mathbf{r}_{K}$ be the $P$-dimensional temporal filters used at the receivers and let $\mathbf{c}$ be the $P$-dimensional temporal signature code used at the transmitter. Note that since the same information is broadcast to all users, only one code is used at the transmitter. Our goal is to design a signature code $\mathbf{c}$ and a set of receive filters $\mathbf{r}_{1}, \ldots, \mathbf{r}_{K}$ that minimizes the transmit power needed to achieve the minimum received SNR requirements. The transmitted signal in its discrete vector form is

$$
\mathbf{x}=b \mathbf{c}
$$

where $b$ is the information bit and $\mathbf{c}$ is the signature code of length $P$. Note that $\mathbf{c}$ is not restricted to be unit norm, and the norm of $\mathbf{c}$ reflects the transmit power. Now let $\mathbf{h}_{k}=\left[h_{k 0}, \ldots h_{k L}\right]^{T}$ be the channel vector of the $k^{t h}$ user sampled at chip interval. The $P$-dimensional received signal vector of the $k^{\text {th }}$ user in its discrete vector form becomes

$$
\mathbf{y}_{k}=\mathbf{H}_{k} \mathbf{x}+\mathbf{n}=b \mathbf{H}_{k} \mathbf{c}+\mathbf{n}
$$

where

$$
\mathbf{H}_{k}=\left[\begin{array}{ccccc}
h_{k 0} & & & & \\
\vdots & \ddots & & 0 & \\
h_{k L} & & \ddots & & \\
& \ddots & & \ddots & \\
0 & & h_{k L} & \cdots & h_{k 0}
\end{array}\right]
$$

is the Toeplitz channel matrix. At the $k$ th receiver, the output of the receive filter $\mathbf{r}_{k}$ is

$$
z_{k}=\mathbf{r}_{k}^{H} \mathbf{y}_{k}=b \mathbf{r}_{k}^{H} \mathbf{H}_{k} \mathbf{c}+\mathbf{r}_{k}^{H} \mathbf{n}
$$

and the optimal detector is $\hat{b}=\operatorname{sign}\left[\operatorname{Re}\left(z_{k}\right)\right]$. The performance of the $k$ th user is measured by the SNR as $\mathrm{SNR}_{k}=\left(1 / \sigma^{2}\right)\left(\operatorname{Re}\left(\mathbf{r}_{k}^{H} \mathbf{H}_{k} \mathbf{c}\right)\right)^{2}$, where $\sigma^{2}$ denotes the noise power. Assuming $\eta_{k}$ to be the target SNR for the $k$ th user, we set up the following optimization problem to find the set of transmit spreading code/receive filters $\mathbf{c}, \mathbf{r}_{1}, \ldots \mathbf{r}_{K}$ that minimizes transmit power while satisfying the SNR constraints of all users:

such that

$$
\mathcal{C}^{\mathrm{opt}} \equiv\left\{\mathbf{c}^{\mathrm{opt}}, \mathbf{r}_{1}^{\mathrm{opt}}, \ldots, \mathbf{r}_{K}^{\mathrm{opt}}\right\}=\arg \min _{\mathbf{c}, \mathbf{r}_{1}, \ldots, \mathbf{r}_{K}} \mathbf{c}^{H} \mathbf{c}
$$

$$
R e\left(\mathbf{r}_{k}^{H} \mathbf{H}_{k} \mathbf{c}\right) \geq \tilde{\eta}_{k} ; \text { and }\left\|\mathbf{r}_{k}\right\|=1, k=1, \ldots, K(5)
$$

where $\tilde{\eta}_{k}=\sqrt{\eta_{k} \sigma^{2}}$ and $\operatorname{Re}(x)$ denotes the real part of a complex number $x$. The problem in the form of (5) requires joint optimization of both transmit signature code $\mathbf{c}$ and receiver filters $\mathbf{r}_{1}, \ldots, \mathbf{r}_{K}$. However, we know that for a given signature code $\mathbf{c}$, the receiver that maximizes the output SNR is a maximum ratio combiner (MRC) $\mathbf{r}_{k}=\left(\mathbf{H}_{k} \mathbf{c} /\left\|\mathbf{H}_{k} \mathbf{c}\right\|^{2}\right)$. Since the constraint in (5) is a minimum output SNR constraint, and an MRC receiver filter maximizes the output SNR for a given c, we conjecture that the MRC is the optimal receiver solution to (5), and the joint optimization problem of (5) can be organized into an optimization problem of $\mathbf{c}$ only. This conjecture turns out to be true and a formal proof of the conjecture is found in the following proposition.

Proposition 1: The optimization problem in (5) is equivalent to

$$
\mathbf{c}^{\text {opt }}=\arg \min _{\mathbf{c}} \mathbf{c}^{H} \mathbf{c} \quad \text { s.t. } \mathbf{c}^{H} \mathbf{H}_{k}^{H} \mathbf{H}_{k} \mathbf{c} \geq \tilde{\eta}_{k}^{2}
$$


and each receiver is an MRC receiver

$$
\mathbf{r}_{k}^{\mathrm{opt}}=\frac{\mathbf{H}_{k} \mathbf{c}^{\mathrm{opt}}}{\left\|\mathbf{H}_{k} \mathbf{c}^{\mathrm{opt}}\right\|} ; \quad k=1, \ldots, K
$$

Proof: See Appendix I.

We have reduced ajoint multivariable optimization problem(5) intoasingle-variableoptimization problem(6). Unfortunately, the quadratic constraint in (6) is a concave constraint. As a result, there is little one can say about either the convergence or the optimality of the solution, if a standard optimization method, for instance, the Lagrange multiplier [8] is directly applied to solve this problem. In this paper, we develop two algorithms that exploit the unique structure of this problem to provide solutions that display better convergence and optimality properties. The first approach is the ILDP algorithm and iteratively optimizes the transmit signature code and receive filters. The second approach is the LP algorithm. Here the concavely constrained quadratic optimization problem (6) is reduced to an approximate LP problem by simultaneous diagonalization of channel matrices.

\section{A. Iterative Optimization-ILDP Algorithm}

In the ILDP algorithm, we derive an iterative procedure from the original joint optimization problem (5), rather than the reduced single-variable optimization problem (6). Observe in (5) that $\mathbf{r}_{1}, \ldots, \mathbf{r}_{k}$ appear only in the constraints, not in the cost function. This structure suggests an iterative solution where the transmit signature code $\mathbf{c}$ and receive filters $\mathbf{r}_{1}, \ldots, \mathbf{r}_{K}$ are solved in an iterative fashion.

\section{ILDP algorithm}

1) Set iteration index $l=1$, start with any set of normalized receive filters $\mathbf{r}_{1}(1), \ldots, \mathbf{r}_{k}(1)$.

2) Solve $\mathbf{c}(l)=\arg \min _{\mathbf{c}} \mathbf{c}^{H} \mathbf{c}$; s.t. $\operatorname{Re}\left(\mathbf{r}_{k}(l)^{H} \mathbf{H}_{k} \mathbf{c}\right) \geq \tilde{\eta}_{k}, k=1, \ldots, K$. We show below that this can be converted into a least distance programming (LDP) problem [9].

3) Update $\mathbf{r}_{k}(l)$ by setting $\mathbf{r}_{k}(l+1)=$ $\left(\mathbf{H}_{k} \mathbf{c}(l) /\left\|\mathbf{H}_{k} \mathbf{c}(l)\right\|\right)$, the MRC solution.

4) Define $J(l) \equiv \mathbf{c}(l)^{H} \mathbf{c}(l)$. If $\mid J(l+1)-$ $\left.J(l)\right|^{2}<\epsilon$ for some $\epsilon>0$, stop; otherwise set $l=l+1$ and go to Step 2).

Note that the sequence of cost function values $J(l)$ always converges. To show this, observe that $\left\{\mathbf{c}(l), \mathbf{r}_{1}(l), \ldots, \mathbf{r}_{K}(l)\right\}$ and $\left\{\mathbf{c}(l+1), \mathbf{r}_{1}(l), \ldots, \mathbf{r}_{K}(l)\right\}$ are both feasible solutions to (5), but Step 2) of the algorithm guarantees that $\mathbf{c}(l+1)$ is the optimal solution given $\left\{\mathbf{r}_{1}(l), \ldots, \mathbf{r}_{K}(l)\right\}$; therefore we have $J(l) \geq J(l+1) \geq 0$. Consequently the sequence of cost function values converges to a limit by the monotone convergence theorem [10].

Iterative algorithms similar to the ILDP algorithm developed in this paper are quite commonly used for solving joint optimization problems. A classical example is the Lloyd-Max algorithm [11] for deriving the optimal quantization solution. While intuitive and easy to implement, the major limitation of such iterative approaches is that in general they converge to a local minimum, not the global minimum.

1) LDP Algorithm: We now focus on Step 2) of the ILDP algorithm. In this step, the transmit spreading code is optimized assuming known receive filters. We repeat the problem in Step 2)

$$
\mathbf{c}^{\text {opt }}=\arg \min _{\mathbf{c}} \mathbf{c}^{H} \mathbf{c} \quad \text { s.t. } \operatorname{Re}\left(\mathbf{r}_{k}^{H} \mathbf{H}_{k} \mathbf{c}\right) \geq \tilde{\eta}_{k}
$$

for $k=1, \ldots, K$. Now let $\mathbf{a}_{k}^{H}=\mathbf{r}_{k}^{H} \mathbf{H}_{k}$ with $\mathbf{A}^{H}=$ $\left[\mathbf{a}_{1}, \ldots, \mathbf{a}_{K}\right]$ and $\boldsymbol{\eta}=\left[\tilde{\eta}_{1}, \ldots, \tilde{\eta}_{K}\right]^{T}$ to rewrite (8) as

$$
\mathbf{c}^{\mathrm{opt}}=\arg \min _{\mathbf{c}} \mathbf{c}^{H} \mathbf{c} \quad \text { s.t. } \operatorname{Re}(\mathbf{A c}) \geq \boldsymbol{\eta} .
$$

We now formulate this optimization problem in real-valued space. Let $\mathbf{c}=\mathbf{c}_{r}+j \mathbf{c}_{i}$ and $\mathbf{A}=\mathbf{A}_{r}+j \mathbf{A}_{i}$ where the subscripts $r$ and $i$ denote real and imaginary parts, respectively. We further define $\mathbf{d}=\left[\mathbf{c}_{r}^{T}, \mathbf{c}_{i}^{T}\right]^{T}$ and $\mathbf{B}=\left[\mathbf{A}_{r},-\mathbf{A}_{i}\right]$. Note that $\mathbf{d}$ has dimension of $2 P \times 1$ while $\mathbf{B}$ has dimension $K \times 2 P$. Problem (9) can be equivalently written as

$$
\mathbf{d}^{\mathrm{opt}}=\arg \min _{\mathbf{d}} \mathbf{d}^{T} \mathbf{d} \quad \text { s.t. } \mathbf{B d} \geq \boldsymbol{\eta} .
$$

The optimization problem (10) is termed an LDP problem [9]. It is easy to see that at least one feasible solution [a feasible solution is a vector $\mathbf{d}$ that satisfies the constraint in (10)] exists when $K \leq 2 P$. For instance, the minimum norm solution $\mathbf{d}=\mathbf{B}^{T}\left(\mathbf{B B}^{T}\right)^{-1} \tilde{\boldsymbol{\eta}}$ corresponding to the equality constraint is one of the feasible solutions for (10). Moreover, within the set of feasible solutions for (10), many local minimums may exist. However, the following proposition establishes that all local minimums have the same performance.

Proposition 2: Every local minimum of (10) is a global minimum. A vector $\mathbf{d}$ is a solution to (10) if and only if there exist Lagrange multipliers $\boldsymbol{\lambda}=\left[\lambda_{1}, \ldots, \lambda_{K}\right]^{T} \in \mathcal{R}_{+}^{K}$ such that ${ }^{1}$

$$
2 \mathbf{d}-\mathbf{B}^{T} \boldsymbol{\lambda}=0 \quad \text { and } \quad \boldsymbol{\lambda}^{T}(\mathbf{B d}-\tilde{\boldsymbol{\eta}})=0 .
$$

Proof: It is easy to see that the cost function in (10) is convex and the constraint is linear. By the Kuhn-Tucker necessity theorem [12], (11) holds at a local minimum. However, the Kuhn-Tucker sufficiency theorem dictates that if (11) holds, it is a global minimizer. Therefore, any solution to (11) yields the global minimum of (10).

Except for several special cases, a closed-form solution for Lagrange multipliers in (11) is difficult to obtain. An efficient iterative algorithm to find the Lagrange multipliers is presented in [9].

\section{B. Approximate Optimization-LP Algorithm}

The major limitations of the ILDP algorithm we developed in the previous section include a) the computational complexity can be high if the number of iterations becomes large and $b$ ) convergence to a global optimum is not guaranteed. In this section, we develop an approximate LP algorithm that is more computationally efficient and that always converges to a global optimum. As we elaborate later, the approximation comes from the step where we approximate the Toeplitz channel matrices with circulant matrices.

\footnotetext{
${ }^{1} \mathbf{x} \in \mathcal{R}_{+}^{N}$ means that all elements of $\mathbf{x}$ are real and nonnegative
} 
We start from the simplified single-variable problem (6). As a consequence of the concave constraint in (6), multiple local minima may exist and iterative algorithms may be trapped in a local minimum, if the starting point is not well selected. Nevertheless, we show in the following proposition that due to the Toeplitz structure of the channel matrices, they can be approximated by circulant matrices which may be simultaneously diagonalized, and therefore we can transform the problem in (6) into a well-studied LP problem. Furthermore, as a property of the LP solution, every local minimum of (6) is a global minimum.

Proposition 3: The problem in (6) can be reduced into an LP problem of the form

$$
\begin{aligned}
& \mathbf{u}^{*}=\arg \min _{\mathbf{u}} \sum_{i=1}^{P} u_{i} ; \text { s.t. } \\
& \sum_{i=1}^{P} u_{i}\left\|\tilde{h}_{k i}\right\|^{2} \geq \tilde{\eta}_{k}^{2} ; \text { and } u_{i} \geq 0, k=1, \ldots, K .
\end{aligned}
$$

Proof: With the previously mentioned assumption $P \gg$ $L$, the Toeplitz matrix $\mathbf{H}_{k}$ can be well approximated by a circulant matrix, that is

$$
\mathbf{H}_{k} \approx\left[\begin{array}{ccccc}
h_{k 0} & 0 & h_{k l} & \cdots & h_{k 1} \\
\vdots & \ddots & 0 & \ddots & \vdots \\
h_{k L} & & \ddots & 0 & h_{k l} \\
& \ddots & & \ddots & 0 \\
0 & & h_{k L} & \cdots & h_{k 0}
\end{array}\right]_{P \times P} .
$$

Furthermore, since all circulant matrices are diagonalized by the discrete Fourier transform (DFT) matrix [13], we have $\mathbf{H}_{k} \approx$ $\mathbf{D} \tilde{\mathbf{H}}_{k} \mathbf{D}^{H}$, where

$$
\mathbf{D}=\left[\begin{array}{cccc}
1 & 1 & \ldots & 1 \\
1 & e^{j\left(\frac{2 \pi}{P}\right)} & & e^{j\left(\frac{2 \pi}{P}\right)(P-1)} \\
\vdots & \vdots & & \vdots \\
1 & e^{j\left(\frac{2 \pi}{P}\right)(P-1)} & \ldots & e^{j\left(\frac{2 \pi}{P}\right)(P-1)^{2}}
\end{array}\right]
$$

is the DFT matrix

$$
\tilde{\mathbf{H}}_{k}=\operatorname{diag}\left(\tilde{\mathbf{h}}_{k}\right)=\left[\begin{array}{lll}
\tilde{h}_{k 1} & & \\
& \ddots & \\
& & \tilde{h}_{k P}
\end{array}\right]
$$

and $\tilde{\mathbf{h}}_{k}=\left[\tilde{h}_{k 1}, \ldots, \tilde{h}_{k P}\right]^{T}=\operatorname{DFT}\left(\left[\mathbf{h}_{k}^{T}, \mathbf{0}^{T}\right]^{T}\right)$ is the zeropadded DFT of the channel vector $\mathbf{h}_{k}=\left[\mathbf{h}_{k 0}, \ldots, \mathbf{h}_{k L}\right]^{T}$. Let $\tilde{\mathbf{c}}=\mathbf{D}^{H} \mathbf{c}$ be the frequency-domain representation of the signature code and (6) be rewritten as

$$
\tilde{\mathbf{c}}^{*}=\arg \min _{\tilde{\mathbf{c}}} \tilde{\mathbf{c}}^{H} \tilde{\mathbf{c}} \quad \text { s.t. } \tilde{\mathbf{c}}^{H} \tilde{\mathbf{H}}_{k}^{H} \tilde{\mathbf{H}}_{k} \tilde{\mathbf{c}} \geq \tilde{\eta}_{k}^{2}
$$

for $k=1, \ldots, K$. The above equation can be organized into the following LP programming problem by defining $u_{i}=\left\|\tilde{c}_{i}\right\|^{2}$ and $\mathbf{u}=\left[u_{1}, \ldots, u_{P}\right]^{T}:$

$$
\begin{aligned}
& \mathbf{u}^{*}=\arg \min _{\mathbf{u}} \sum_{i=1}^{P} u_{i} ; \quad \text { such that } \\
& \sum_{i=1}^{P} u_{i}\left\|\tilde{h}_{k i}\right\|^{2} \geq \tilde{\eta}_{k}^{2}, \text { and } u_{i} \geq 0, k=1, \ldots, K .
\end{aligned}
$$

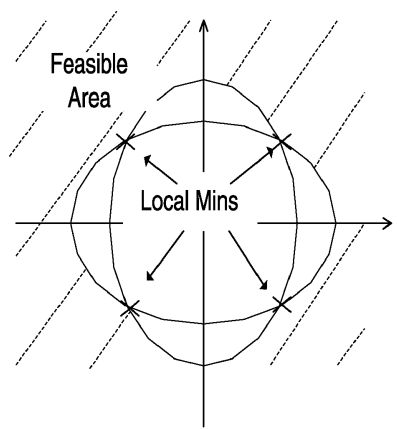

(a)

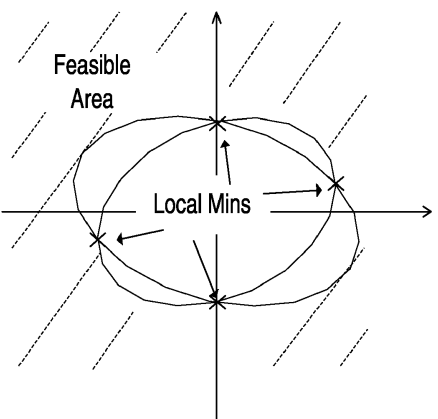

(b)
Fig. 2. Geometric symmetry in the solution space. (a) Solution space with simultaneously diagonalizable channel matrices. (b) Solution space with arbitrary channel matrices.

It might seem odd that we are actually able to convert a nonconvex problem (6) into a convex problem (17) via this change of variable. However, note that this transformation is possible only when the channels are diagonalizable. With this assumption, the solution space of $\mathbf{c}$ exhibits a certain geometric symmetry. Fig. 2 illustrates this symmetry in a two-dimensional solution space when the number of users is also two. Fig. 2(a) assumes that the channel matrices are simultaneously diagonalized while Fig. 2(b) does not. In each figure the feasible solution area is shaded outside the two ellipsoids, which are defined by the constraints in (6). The intersections of the ellipsoids denotes the local minima of the optimization problem (6). Furthermore, note that in Fig. 2(a) the ellipsoids are symmetric about the axes. As a result all the local minima in Fig. 2(a) have the same vector norm. And since the norm of the solution completely determines the cost function in the original problem (6), all local minima are also global minima. By reformulating the problem in terms of the norm of $\mathbf{c}$ we obtain a convex problem.

\section{Practical Implementation}

This section discusses the information exchange required to implement these algorithms at the base station (BS) and mobile station (MS). We first show how the optimal receive filters are obtained at the MS side. For ease of exposition, we assume that the optimal transmit signature code $\mathbf{c}^{\mathrm{opt}}$ is known at the transmitter and is used in broadcasting. At the MS side, since the MRC receive filters are given by $\mathbf{r}_{k}=\left(\mathbf{H}_{k} \mathbf{c}^{\text {opt }} /\left\|\mathbf{H}_{k} \mathbf{c}^{\text {opt }}\right\|^{2}\right)$, it seems that the MS needs to know the BS code $\mathbf{c}^{\mathrm{opt}}$ in order to proceed, which is unrealistic since a reliable communication link between BS to MS is not yet established at this point. However, the MS can consider the BS code as a part of the composite channel $\mathbf{H}_{k} \mathbf{c}^{\text {opt }}$, and therefore obtain the MRC receiver by estimating this composite channel. Note that in an FDD system, since the BS requires feedback of channel estimate $\mathbf{H}_{k}$ from the MS, both $\mathbf{H}_{k}$ and $\mathbf{H}_{k} \mathbf{c}^{\text {opt }}$ have to be estimated at the $k$ th MS. Thus, the BS needs to transmit two sets of training sequences, one set using a known pilot code (for example, $\mathbf{c}=[1,1, \ldots, 1]$ ) for estimating $\mathbf{H}_{k}$, and the other set using the optimal BS code $\mathbf{c}^{\text {opt }}$ for estimating $\mathbf{H}_{k} \mathbf{c}^{\text {opt }}$ at the $k$ th MS.

A more centralized treatment, however, is required to optimize the transmit signature code at the BS side. This is evident since for both ILDP and LP algorithms, all the user chan- 


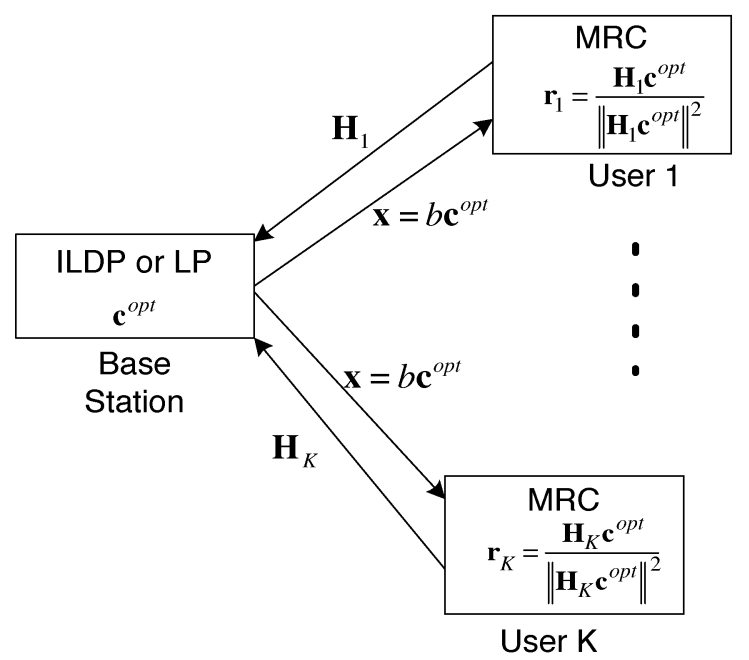

(a)

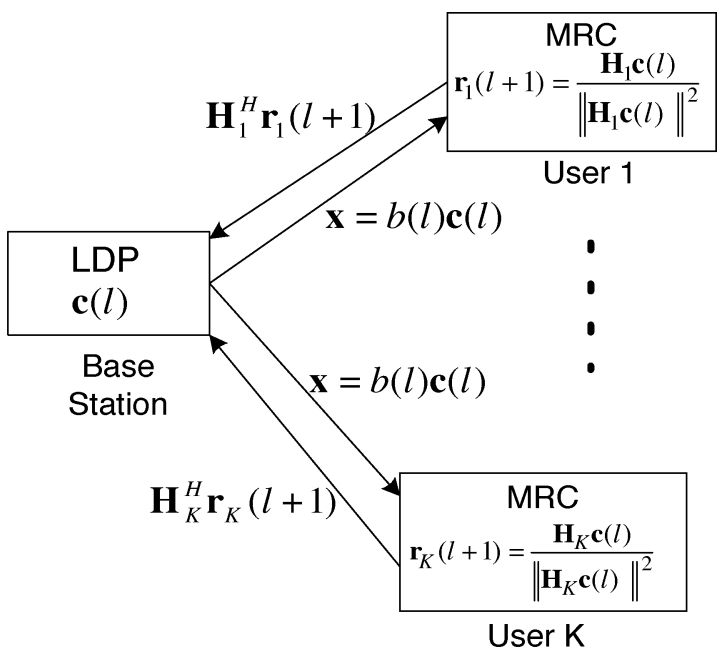

(b)

Fig. 3. Algorithm implementation and flow of information exchange. (a) Implementation of ILDP and LP algorithms at BS and MS in an FDD system and (b) adaptive implementation of ILDP algorithm in an FDD system.

nels $\mathbf{H}_{1}, \ldots, \mathbf{H}_{K}$ are needed to generate the optimal BS code $\mathbf{c}^{\text {opt }}$. The overall flow of information exchange is illustrated in Fig. 3(a). We assume an FDD system and therefore the channel state $\mathbf{H}_{1}, \ldots, \mathbf{H}_{K}$ are fed back from MS to the BS. The BS collects all the channel state and optimizes the signature code $\mathbf{c}^{\text {opt }}$ with the help of either the ILDP or LP algorithm. Finally, the receive filters $\mathbf{r}_{1}, \ldots, \mathbf{r}_{K}$ are separately generated at each MS using an MRC rule.

There is an interesting variation of this implementation that is possible for the ILDP algorithm. The implementation of the ILDP algorithm as shown in Fig. 3(a) represents a "block processing" approach, where the optimal code $\mathbf{c}^{\text {opt }}$ is computed with the iterative algorithm and kept constant for many symbol intervals, before it is recomputed to account for the variations in the channel state. However, in realistic systems, the variation in channel state is usually slow (on the order of ten symbols) over time, thus allowing an alterative "adaptive" implementation of the ILDP algorithm, as shown in Fig. 3(b). In the adaptive implementation, only one iteration of LDP algorithm is carried out within one symbol interval and the convergence to the optimal BS code is achieved over many symbol intervals. In a sense, the adaptive implementation provides a low-complexity solution for tracking the channel state of all the users, even though the rates of the channel variations may differ significantly for users travelling at different speeds. Note that in the adaptive implementation, the necessary feedback from the $k$ th MS to BS becomes $\mathbf{H}_{k}^{H} \mathbf{r}_{k}$ instead of channel matrix $\mathbf{H}_{k}$.

\section{JoINT SPACE-TIME DESIGN}

In this section we study the case where multiple transmit antennas are incorporated in the system. We show that the ILDP algorithm is still applicable for space-time optimization. However, the LP algorithm cannot be applied directly since the channels of different users in the spatial dimension do not share a common eigen structure and thus cannot be jointly diagonalized. To circumvent this limitation, we develop a method of in- tegrating the LP algorithm with the space-time block codes described in [6] and [7] and term it STC-LP algorithm.

Similar to (5), we set up the joint transmit/receive optimization problem as

$$
\mathcal{C}^{\mathrm{opt}} \equiv\left\{\mathbf{c}^{\mathrm{opt}}, \mathbf{r}_{1}^{\mathrm{opt}}, \ldots, \mathbf{r}_{K}^{\mathrm{opt}}\right\}=\arg \min _{\mathbf{c}, \mathbf{r}_{1}, \ldots, \mathbf{r}_{K}} \mathbf{c}^{H} \mathbf{c}
$$

such that

$$
\operatorname{Re}\left(\mathbf{r}_{k}^{H} \mathbf{H}_{k} \mathbf{c}\right) \geq \tilde{\eta}_{k} ; \text { and }\left\|\mathbf{r}_{k}\right\|=1, k=1, \ldots, K .
$$

Note that in this case $\mathbf{c}=\left[\mathbf{c}_{1}^{T}, \ldots, \mathbf{c}_{N}^{T}\right]^{T}$ is a space-time transmit signature code of dimension $N P \times 1$, with $N$ being the number of transmitter antennas and $\mathbf{c}_{n}$ being the spreading code at $n^{\text {th }}$ antenna. Accordingly, $\mathbf{H}_{k}=\left[\mathbf{H}_{k 1}, \ldots, \mathbf{H}_{k N}\right]$ becomes a $P \times N P$ matrix, where $\mathbf{H}_{k n}$ is the channel matrix coupling the $n^{t h}$ transmit antenna and the $k$ th receiver. Note that although each $\mathbf{H}_{k n}$ has a Toeplitz structure, the overall channel matrix $\mathbf{H}_{k}$ does not.

\section{A. ILDP Algorithm}

It is evident that (18) is similar to (5) and thus can be solved by the same ILDP algorithm we developed in Section II-A with slight modifications to $\mathbf{c}$ and $\mathbf{H}_{k}$. Again, in general this iterative algorithm converges to a local minimum of the optimization problem.

\section{B. STC-LP Algorithm}

In Section II-B, it is shown that due to the approximate circulant structure of the channel matrices in the temporal dimension, they can be simultaneously diagonalized. This enables the original problem (5) to be transformed into the convex LP problem given in (17). This transformation, however, is not possible in a space-time channel since the overall space-time channel matrices $\mathbf{H}_{k}$ do not share a common eigen structure in the spatial dimension. As a result, the LP algorithm is not directly applicable in this case.

In this section, we explore a different path to obtain a computationally efficient, yet suboptimal, LP algorithm by incorpo- 
rating space-time block codes from orthogonal design, as described in [6] and [7]. Instead of sending out the same bit across all the transmit antennas at a certain time slot, we consider a block of $N$ bits and send them out over $N$ time slots in a pattern that is known to both the transmitter and receivers. Note that $N$ is the number of antennas at the transmitter. The result of this operation is the introduction of "controlled" self-interference at each time slot, which can be eliminated after all the $N$ bits are received. After the elimination of the self-interference, the detection of each bit in the sequence can be reduced to an LP problem similar to the one that we solved in the time-only optimization. The "controlled" self-interference, however, also results in a slight performance degradation compared with the ILDP algorithm.

For ease of exposition we assume in the discussion below that the number of transmit antennas is two. The extension to an arbitrary number of antennas requires a more sophisticated orthogonal design of space-time block code as described in [6]. In the case of two transmit antennas, the space-time block code pairs up two bits $b_{1}$ and $b_{2}$ and transmits them over two time slots. With the so-called orthogonal design [6], we assure that the controlled self-interference can be eliminated by jointly processing the two time slots at the receiver. To see this, let $\mathbf{c}_{1}, \mathbf{c}_{2}$ be the transmit signature codes at the two transmit antennas and let

$$
\mathbf{B}=\left[\begin{array}{cc}
b_{1} & b_{2} \\
-b_{2} & b_{1}
\end{array}\right]
$$

be the orthogonal code matrix. At time slot $t \in\{1,2\}$, the $n$th $(n \in\{1,2\})$ antenna will transmit information bit $(\mathbf{B})_{t n}$, the bit in $t$ th row and $n$th column of matrix $\mathbf{B}$. Let $\mathbf{H}_{k 1}, \mathbf{H}_{k 2}$ be the $P \times P$ Toeplitz matrices that couple receiver $k$ with transmit antennas 1 and 2 . We further assume that the channel is constant over the two time slots and denote the received vector at the two time slots by $\mathbf{y}_{k 1}$ and $\mathbf{y}_{k 2}$. Stacking up $\mathbf{y}_{k 1}$ and $\mathbf{y}_{k 2}^{*}$ and defining $\mathbf{y}_{k} \equiv\left[\mathbf{y}_{k 1}, \mathbf{y}_{k 2}\right]^{T}$, we get

$$
\begin{aligned}
\mathbf{y}_{k} & =\left[\begin{array}{cc}
\mathbf{H}_{k 1} \mathbf{c}_{1} & \mathbf{H}_{k 2} \mathbf{c}_{2} \\
\left(\mathbf{H}_{k 2} \mathbf{c}_{2}\right)^{*} & \left(-\mathbf{H}_{k 1} \mathbf{c}_{1}\right)^{*}
\end{array}\right]\left[\begin{array}{l}
b_{1} \\
b_{2}
\end{array}\right]+\left[\begin{array}{l}
\mathbf{n}_{1} \\
\mathbf{n}_{2}
\end{array}\right] \\
& \equiv \mathbf{R}_{k} \mathbf{b}+\mathbf{n} .
\end{aligned}
$$

In (20), the "controlled" self-interference is represented by the cross terms in matrix $\mathbf{R}_{k}$. However, since each $\mathbf{R}_{k}$ is an orthogonal matrix, we can cancel the self-interference by multiplying with $\mathbf{R}_{k}^{H}$

$$
\mathbf{z}_{k}=\left[\begin{array}{c}
z_{k 1} \\
z_{k 2}
\end{array}\right] \equiv \mathbf{R}_{k}^{H} \mathbf{y}_{k}=\mathcal{E}_{s}\left[\begin{array}{l}
b_{1} \\
b_{2}
\end{array}\right]+\tilde{\mathbf{n}}
$$

where

$$
\begin{aligned}
\mathcal{E}_{s} & \equiv\left(\mathbf{c}_{1}{ }^{H} \mathbf{H}_{k 1}^{H} \mathbf{H}_{k 1} \mathbf{c}_{1}+\mathbf{c}_{2}^{H} \mathbf{H}_{k 2}^{H} \mathbf{H}_{k 2} \mathbf{c}_{2}\right) \\
\tilde{\mathbf{n}} & \sim \mathcal{N}\left(0, \mathcal{E}_{s} \sigma^{2} \mathbf{I}_{2 \times 2}\right) .
\end{aligned}
$$

Consequently, the maximum likelihood detectors for $b_{1}$ and $b_{2}$ are given by $\hat{b}_{1}=\operatorname{sign}\left[\operatorname{Re}\left(z_{k 1}\right)\right], \hat{b}_{2}=\operatorname{sign}\left[\operatorname{Re}\left(z_{k 2}\right)\right]$. The corresponding SNRs are

$$
\begin{aligned}
\mathrm{SNR}_{k} & =\mathrm{SNR}_{k 1}=\mathrm{SNR}_{k 2}=\frac{\mathcal{E}_{s}}{\sigma^{2}} \\
& =\frac{1}{\sigma^{2}}\left(\mathbf{c}_{1}{ }^{H} \mathbf{H}_{k 1}^{H} \mathbf{H}_{k 1} \mathbf{c}_{1}+\mathbf{c}_{2}^{H} \mathbf{H}_{k 2}^{H} \mathbf{H}_{k 2} \mathbf{c}_{2}\right) .
\end{aligned}
$$

Hence an optimization problem similar to (6) can be formulated to minimize the overall transmit power while satisfying the SNR requirement of all users

$$
\begin{array}{r}
{\left[\mathbf{c}_{1}^{\mathrm{opt}}, \mathbf{c}_{2}^{\mathrm{opt}}\right]=\arg \min _{\mathbf{c}_{1}, \mathbf{c}_{2}} \mathbf{c}_{1}^{H} \mathbf{c}_{1}+\mathbf{c}_{2}^{H} \mathbf{c}_{2} \quad \text { such that }} \\
\mathbf{c}_{1}^{H} \mathbf{H}_{k 1}^{H} \mathbf{H}_{k 1} \mathbf{c}_{1}+\mathbf{c}_{2}^{H} \mathbf{H}_{k 2}^{H} \mathbf{H}_{k 2} \mathbf{c}_{2} \geq \tilde{\eta}_{k}^{2} ; k=1, \ldots, K .
\end{array}
$$

We solve this problem in the frequency domain by letting $\mathbf{H}_{k 1}=\mathbf{D} \tilde{\mathbf{H}}_{k 1} \mathbf{D}^{H}, \mathbf{H}_{k 2}=\mathbf{D} \tilde{\mathbf{H}}_{k 2} \mathbf{D}^{H}$, and $\tilde{\mathbf{c}}_{1}=\mathbf{D}^{H} \mathbf{c}_{1}$, $\tilde{\mathbf{c}}_{2}=\mathbf{D}^{H} \mathbf{c}_{2}$. Furthermore, let $\tilde{\mathbf{c}}=\left[\tilde{\mathbf{c}}_{1}^{T}, \tilde{\mathbf{c}}_{2}^{T}\right]^{T}$ be the concatenated frequency-domain space-time signature code and let

$$
\tilde{\mathbf{H}}_{k}=\left[\begin{array}{cc}
\tilde{\mathbf{H}}_{k 1} & \mathbf{0} \\
\mathbf{0} & \tilde{\mathbf{H}}_{k 2}
\end{array}\right]
$$

be the overall frequency domain channel matrix where $\tilde{\mathbf{H}}_{k 1}$ and $\tilde{\mathbf{H}}_{k 2}$ are the frequency-domain channel matrix for antennas 1 and 2, respectively, as defined in (15). With these definitions the optimization problem (22) may be rewritten as

$$
\begin{array}{r}
\tilde{\mathbf{c}}^{\text {opt }}=\arg \min _{\tilde{\mathbf{c}}} \tilde{\mathbf{c}}^{H} \tilde{\mathbf{c}} \quad \text { such that } \\
\tilde{\mathbf{c}}^{H} \tilde{\mathbf{H}}_{k}^{H} \tilde{\mathbf{H}}_{k} \tilde{\mathbf{c}} \geq{\tilde{\eta_{k}}}^{2} ; \quad k=1, \ldots, K
\end{array}
$$

which is in exactly the same form as (16). Therefore, by Proposition 3, it can be converted into an LP problem analogous to (17).

\section{SPACE-ONLY DESIGN}

In previous sections we designed optimal space-time transmit signature codes and receive filters for a selective broadcast system. In this section we optimize only over the spatial dimension, that is, we design transmit antenna weights assuming a fixed signature code at the transmitter and fixed filters at the receivers. This problem is relevant if the temporal signature code in a system is predetermined and not subject to any optimization. Note that in this section we assume a flat fading channel $(L=1)$ to simplify the discussion. Now let c be the unit-norm transmit spreading code. Since $L=1$, it is easily shown that $\mathbf{r}_{k}=\mathbf{c}$ is the MRC solution at the $k$ th receiver. Let $\mathbf{w}=\left[w_{1}, \ldots, w_{N}\right]^{T}$ be the weight vector at the transmit antennas. The transmitted signal at the $n$th antenna is $\mathbf{x}_{n}=b w_{n} \mathbf{c}$, and the received signal of the $k^{t h}$ user is

$$
\mathbf{y}_{k}=\sum_{n=1}^{N} \mathbf{x}_{n} h_{k n}+\mathbf{n}_{k}=b \mathbf{w}^{T} \mathbf{h}_{k} \mathbf{c}+\mathbf{n}_{k} .
$$

Projecting the received signal onto the code $\mathbf{c}$, we get $z_{k}=$ $b \mathbf{w}^{T} \mathbf{h}_{k}+\tilde{n}_{k}$, where $\tilde{n}_{k}=\mathbf{c}^{H} \mathbf{n}_{k}$ is independent identically distributed with variance $\sigma^{2}$ for $k=1, \ldots, K$. Similar to the approach adopted in the previous sections, we pose the following optimization problem to minimize the transmit power while satisfying the minimum received SNRs:

$$
\begin{aligned}
& \mathbf{w}^{\text {opt }}=\arg \min _{\mathbf{w}} \mathbf{w}^{H} \mathbf{w} \quad \text { such that } \\
& \mathbf{w}^{H} \mathbf{h}_{k} \mathbf{h}_{k}^{H} \mathbf{w} \geq \tilde{\eta}_{k}^{2} ; \quad k=1, \ldots, K .
\end{aligned}
$$

\section{A. STC-LP and ILDP Algorithms}

The STC-LP algorithm developed for space-time optimization can be applied in this case with minor modifications. Good 
performance is achieved with this algorithm, as will be shown in the simulation results. On the other hand, the ILDP algorithm we developed for the time-only and space-time design cannot be applied to the space-only optimization problem. The reason is that the receive filter is fixed in the space-only optimization problem and the transmitter-receiver iterative procedure is not applicable.

\section{B. Iterative Spatial Diagonalization (ISD) Algorithm}

We now propose an algorithm for obtaining $\mathbf{w}$ that exploits the structure of the spatial optimization problem (26), assuming the number of users $K$ is less than or equal to the number of antennas $N$. We show that this solution is optimal when $N \leq 2$ and is a good suboptimal approximation when $N>2$. First we aim to find a spatial diagonalization matrix $\mathbf{T}$ that satisfies

$$
\mathbf{T H}=\left[\begin{array}{ccc}
\tilde{\eta}_{1} & & \\
\vdots & \ddots & \\
\vdots & & \tilde{\eta}_{K} \\
\vdots & & \vdots \\
0 & \cdots & 0
\end{array}\right] .
$$

Here $\mathbf{H}=\left[\mathbf{h}_{1}, \mathbf{h}_{2}, \ldots, \mathbf{h}_{k}\right]$ is an $N \times K$ matrix whose columns are the channel vectors. Now we define $\mathbf{H}=\mathbf{S D V}^{H}$ to be the singular value decomposition (SVD) of $\mathbf{H}$, and $\mathbf{A}=\operatorname{diag}\left(\left[\tilde{\eta}_{1}, \ldots, \tilde{\eta}_{K}\right]\right)$ to be the diagonal matrix whose diagonal elements are the desired SNRs. It follows that $\mathbf{T}$ can be constructed as

$$
\mathbf{T}=\left[\begin{array}{ll}
\mathbf{A V D}^{-1} & \\
& 0
\end{array}\right] \mathbf{S}^{H} .
$$

Denoting $\mathbf{w}=\mathbf{T}^{H} \tilde{\mathbf{w}}$, it can be shown that (26) reduces to

$$
\begin{aligned}
& \tilde{\mathbf{w}}^{\text {opt }}=\arg \min _{\tilde{\mathbf{w}}} \tilde{\mathbf{w}}^{H} \mathbf{T} \mathbf{T}^{H} \tilde{\mathbf{w}} \\
& \text { such that }\left|\tilde{w}_{k}\right| \geq 1 ; k=1, \ldots, K .
\end{aligned}
$$

We now define $\mathbf{U}=\mathbf{T} \mathbf{T}^{H}, J=\tilde{\mathbf{w}}^{H} \mathbf{T} \mathbf{T}^{H} \tilde{\mathbf{w}}$. Furthermore, we use $\left|\tilde{w}_{i}\right|$ and $\left|U_{i j}\right|$ to denote the amplitudes of complex variables $\tilde{w}_{i}$ and $U_{i j}$, and $\theta_{i}$ and $\phi_{i j}$ to denote the phase angles of $\tilde{w}_{i}$ and $U_{i j}$. We may then write the cost function in the following form:

$$
\begin{aligned}
J(\boldsymbol{\theta}, \hat{\mathbf{w}})= & \sum_{i=1}^{K} U_{i i}\left|\tilde{w}_{i}\right|^{2} \\
& +2 \sum_{i=1}^{K} \sum_{j=1}^{i-1}\left|U_{i j}\right|\left|\tilde{w}_{i}\right|\left|\tilde{w}_{j}\right| \cos \left(\theta_{i}-\theta_{j}-\phi_{i j}\right)
\end{aligned}
$$

where $\boldsymbol{\theta}=\left[\theta_{1}, \ldots, \theta_{K}\right]$ and $\hat{\mathbf{w}}=\left[\left|\tilde{w}_{1}\right|, \ldots\left|\tilde{w}_{K}\right|\right]^{T}$. Since $\cos (\cdot)$ is bounded between -1 and 1 , it will be ideal if we can find a set of $\theta_{i}$ such that all the $\cos \left(\theta_{i}-\theta_{j}-\phi_{i j}\right)=-1$ for any $i, j$, which means $\theta_{i}-\theta_{j}+\phi_{i j}=\pi, \forall i$ and $j<i$. However, if one write this set of equations into a matrix form for $K=3$

$$
\mathbf{A} \boldsymbol{\theta} \equiv\left[\begin{array}{ccc}
1 & -1 & 0 \\
1 & 0 & -1 \\
0 & 1 & -1
\end{array}\right]\left[\begin{array}{l}
\theta_{1} \\
\theta_{2} \\
\theta_{3}
\end{array}\right]=\left[\begin{array}{l}
\pi-\phi_{12} \\
\pi-\phi_{13} \\
\pi-\phi_{23}
\end{array}\right] \equiv \boldsymbol{\phi}
$$

it is easy to see that $\mathbf{A}$ is of rank 2 , and therefore no solution exists for $K>2$. The best approximation one may get is a pseudosolution $\boldsymbol{\theta}^{*}=\mathbf{A}^{+} \boldsymbol{\phi}$ where $\mathbf{A}^{+}$denotes the pseudoinverse of A.

Since a simple closed-form solution can not be obtained, we again resort to an iterative process. Letting $\boldsymbol{\theta}^{*}$ be an initial estimation and let $d_{i j}=\cos \left(\theta_{i}^{*}-\theta_{j}^{*}-\phi_{i j}\right)$, we write (28) as

$$
\hat{\mathbf{w}}^{\text {opt }}=\min _{\hat{\mathbf{w}}} \hat{\mathbf{w}}^{T} \mathbf{Z} \hat{\mathbf{w}} \quad \text { s.t. } \hat{\mathbf{w}} \geq \mathbf{1}
$$

where $\mathbf{1}=[1, \ldots, 1]^{T}$ and $\mathbf{Z}$ is a positive semidefinite matrix with elements $Z_{i i}=U_{i i}, Z_{i j}=Z_{j i}=\left|U_{i j}\right| d_{i j}$. Equation (31) defines a least squares with inequality constraint (LSI) problem. It is similar to the LDP problem we discussed previously and can be solved by the LSI algorithm described in [9]. Once $\hat{\mathbf{w}}$ is obtained by the LSI algorithm, we iterate by solving (29) for the phase angles $\theta_{1}, \ldots, \theta_{K}$ using the steepest decent algorithm to minimize $J=J\left(\theta_{1}, \ldots \theta_{K}\right)$. This iterative approach is summarized below as an ISD algorithm.

\section{Iterative Spatial Diagonalization (ISD) Algorithm}

1) Set iteration index $l=1$, get an initial estimate of

$\boldsymbol{\theta}(1)=\left[\theta_{1}(1), \ldots, \theta_{K}(1)\right]^{T}$ by solving (30).

2) For given $\boldsymbol{\theta}(l)$, solve (31) using

LSI algorithm to update $\hat{\mathbf{w}}(l)$.

3) For given $\hat{\mathbf{w}}(l)$, obtain $\boldsymbol{\theta}(l+1)$ by minimizing (29) with the steepest descent algorithm.

4) If $|J(l+1)-J(l)|^{2}<\epsilon$ for some $\epsilon>0$, stop; otherwise set $l=l+1$ and go to Step 2).

\section{Simulation Results}

In this section we evaluate the effectiveness of the algorithms developed in this paper via numerical simulations. We follow the classification adopted in the previous sections and divide the simulation results into time-only, space-time, and space-only design subsections. The results show a $3.0 \mathrm{~dB}$ transmit power reduction with the ILDP or LP algorithm, compared with a simple broadcast scheme. The transmit power can be further reduced by $2-2.5 \mathrm{~dB}$ via ILDP or STC-LP algorithm when the number of transmit antennas is increased from one to two. It is also shown that the algorithms are robust when the estimated channel coefficients are corrupted by estimation errors or feedback delays.

\section{A. Time-Only Design}

In this section we evaluate the performance of the ILDP and LP algorithms for temporal optimization. The transmit signature code is assumed to have length $P=16$ and the multipath spread of the channel is assumed to be $L=4$ chips long. The channel coefficient vector $\mathbf{h}_{k}$ for user $k$ is assumed to be complex Gaussian $\mathbf{h}_{k} \sim \mathcal{N}\left(\mathbf{0},(1 / L+1) \mathbf{I}_{L+1}\right)$, where $\mathbf{I}_{L+1}$ is the 


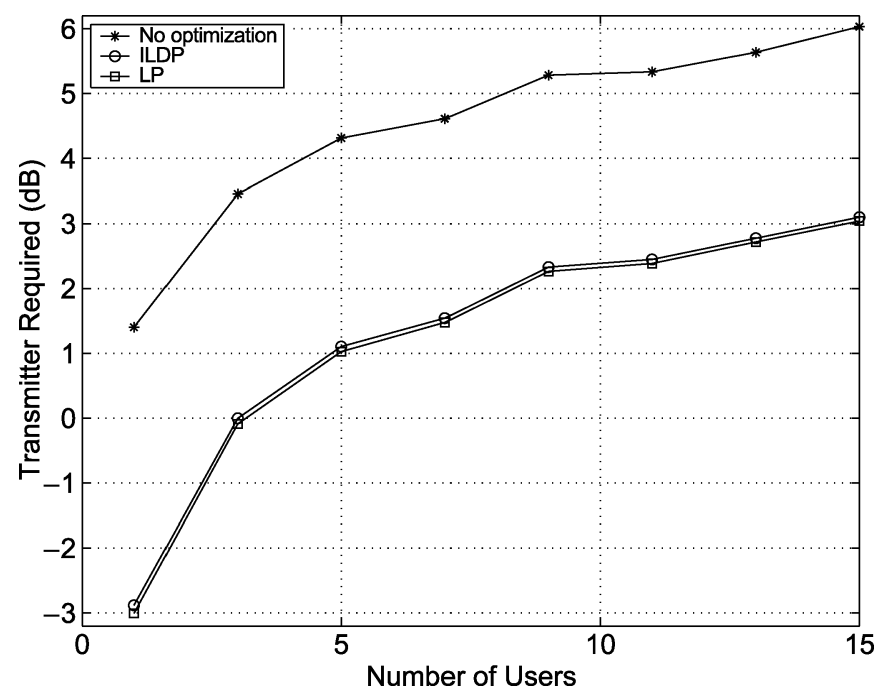

Fig. 4. Comparison of algorithms for temporal optimization. $L=4, P=16$. The ILDP and LP algorithms hold a similar $3 \mathrm{~dB}$ gain compared with nonoptimal broadcast.

$L+1 \times L+1$ identity matrix. Fig. 4 illustrates the dependence of required transmit power on the number of users for different algorithms. The curve "No Optimization" in Fig. 4 stands for the simple broadcast scheme described in the introduction $\left(P_{\text {total }}=\max _{k}\left\{P_{k}\right\}\right)$. It is observed that both the ILDP and LP algorithms have a significant $3 \mathrm{~dB}$ gain against the simple nonoptimal broadcast algorithm. It is also evident in Fig. 4 that ILDP and LP curves almost overlap with each other. The small discrepancy between the two curves is mainly due to the circulant approximation of the Toeplitz channel matrix. Fig. 5 further illustrates the convergence of ILDP algorithm. In most realizations, ILDP converges in 10-20 iterations.

1) Sensitivity Against Channel Estimate Delays: It is assumed in the algorithms that the channel coefficients are known at the transmitter via channel estimation or feedback. However, in realistic situations these channel coefficients can be erroneous due to estimation errors or feedback delays. To evaluate the robustness of the algorithms, we study the performance of the LP algorithm given delayed channel estimates. The autoregressive process of order one (AR-1) model used in [14] is adopted for channel state evolution. Define $\hat{h}_{k l}$ as the delayed version of $h_{k l}$ and assume that $\left\{h_{k l}\right\}$ evolve independently, we have for $l=0, \ldots, L$ and $k=1, \ldots, K$

$$
\begin{aligned}
h_{k l} & =\rho \hat{h}_{k l}+\theta_{k l} \\
E\left[\hat{h}_{k l}^{*} \theta_{k l}\right] & =0 \\
\theta k l & \sim \mathcal{N}\left[0,\left(1-\rho^{2}\right) \times E\left|h_{k l}\right|^{2}\right]
\end{aligned}
$$

where $\rho=J_{0}\left(2 \pi f_{d} D\right)$, with $J_{0}(x)$ being the zero-order Bessel function of the first kind. $f_{d}$ is the Doppler spread in hertz and $D$ is the amount of delay in seconds. It is obvious that the correlation between $h_{k l}$ and $\hat{h}_{k l}$ decreases with a larger Doppler spread $f_{d}$ (higher vehicle speed) and longer feedback delay $D$.

The LP algorithm is used to design the transmit signature $\mathbf{c}$ and receive filters $\left\{\mathbf{r}_{k}\right\}$ based on $\hat{h}_{k l}$ instead of $h_{k l}$. The impact of the channel estimate delay is manifested in the amount of additional power needed to satisfy the SNR requirements for the

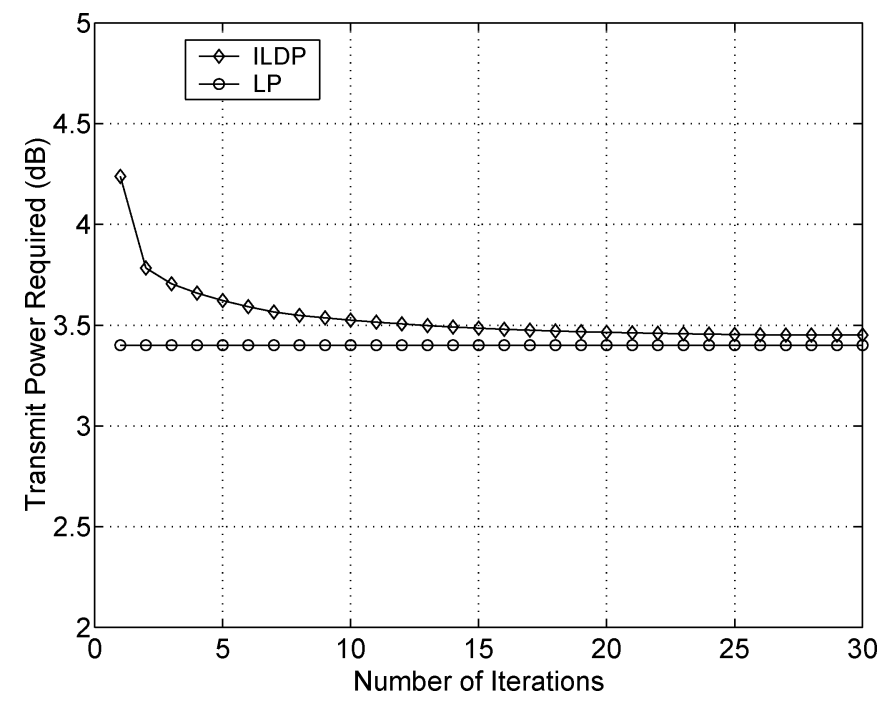

Fig. 5. Convergence of ILDP to LP. $L=4, P=16$. Convergence usually takes 10-20 iterations.

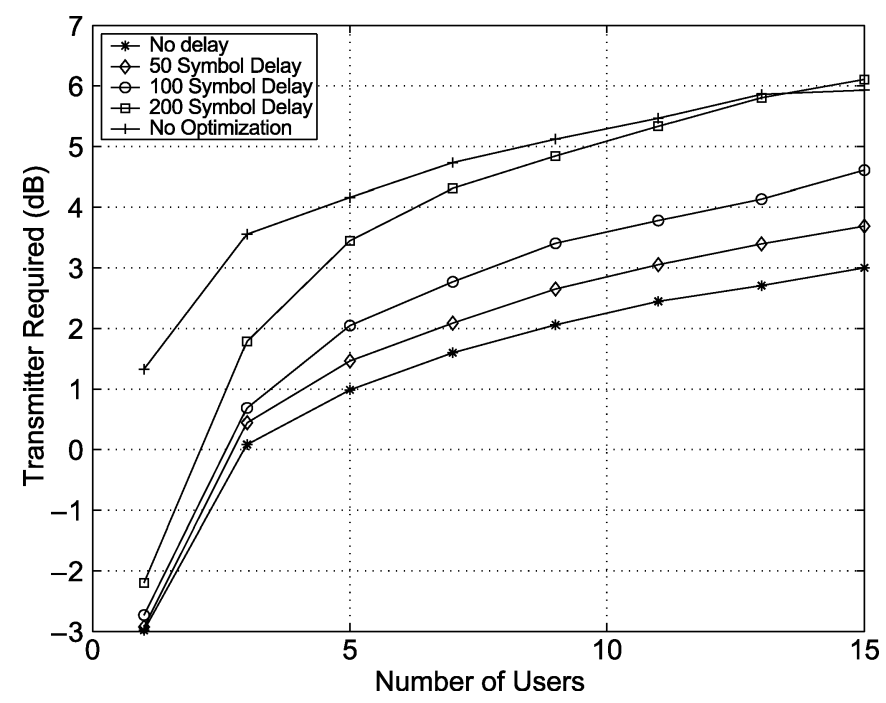

Fig. 6. Robustness of the algorithm with delayed channel estimates at the transmitter. $L=4, P=16$. The performance loss is insignificant with a reasonably small delay (less than 50 symbols).

users. The parameters of the numerical simulations are: speed of all users $50 \mathrm{~km} / \mathrm{h}$, carrier frequency $2 \mathrm{GHz}$, data symbol rate $100 \mathrm{kHz}$, and the feedback delay is set at 50,100 , and $200 \mathrm{sym}-$ bols, respectively. Fig. 6 shows the comparison of the transmission power needed with these three delay parameters. With 50 symbol delay, the additional power needed is only about $0.6 \mathrm{~dB}$. However, with a 200 symbol delay, the performance of the LP algorithm almost degrades to the level of simple broadcast algorithm. Therefore, it is critical to have a reasonably fast feedback mechanism in order to reap the benefits of optimal broadcast algorithms.

2) Peak to Average Power Ratio: Unlike a conventional binary spreading code, the BS signature code we designed takes arbitrary complex values. One practical concern is whether this complex signature code will result in a high peak-to-average ratio (PAR) at the transmitter. As shown in [15], the PAR is a probabilistic notion and is usually described by its complementary cumulative distribution function (CCDF). The closed-form 


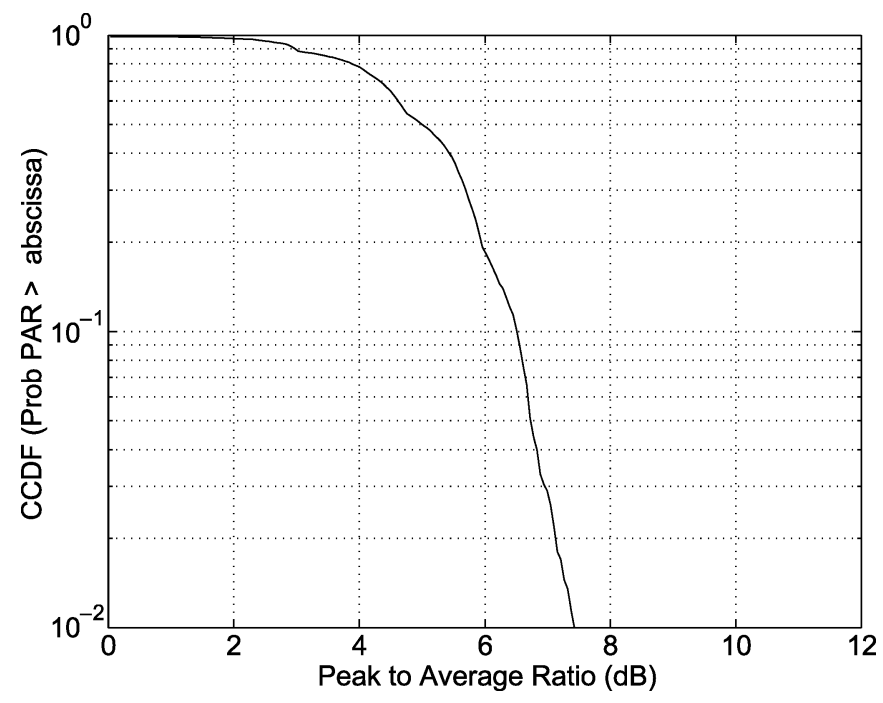

Fig. 7. Peak to average power ratio at the transmitter for $P=16, L=4$, $K=9$. The probability of PAR exceeding $6.5 \mathrm{~dB}$ is about $10 \%$.

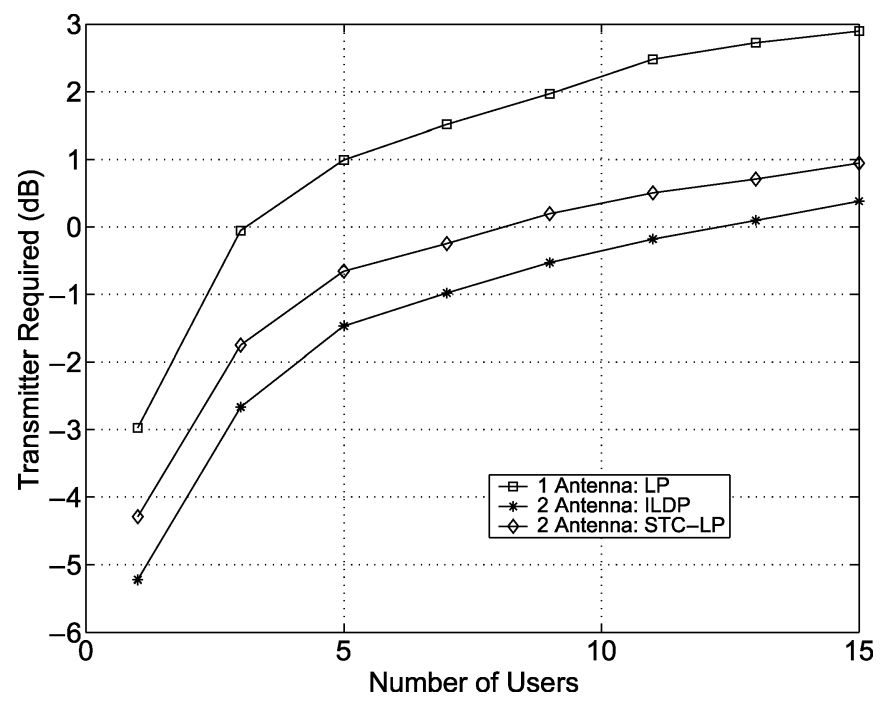

Fig. 8. Space-time optimization: $N=2, L=4, P=16$. A $2-2.5 \mathrm{~dB}$ total transmit power is saved with the addition of another transmit antenna.

CCDF of PAR in our broadcast problem is hard to obtain, and we resort to numerical simulations to illustrate the behavior of PAR. In Fig. 7, the CCDF is simulated for a nine-user system with spreading code length $P=16$ and channel length $L=4$. In this case, the probability of PAR exceeding $6.5 \mathrm{~dB}$ is only about $10 \%$, which is fairly reasonable for a realistic system, considering that the probability of PAR exceeding $11 \mathrm{~dB}$ is about $10 \%$ for a typical 256 subcarrier orthogonal frequency-division multiplexing system [15].

\section{B. Space-Time Design}

Both ILDP and STC-LP algorithms are evaluated for $N=2$ transmitter antennas and the results are shown in Fig. 8. The results from the LP algorithm for the single antenna case are also given as a reference. The other parameters stay the same at $L=4$ and $P=16$. It is observed that with $N=2$ spatial dimensions, both ILDP and STC-LP algorithms perform significantly better than the single antenna LP algorithm. At

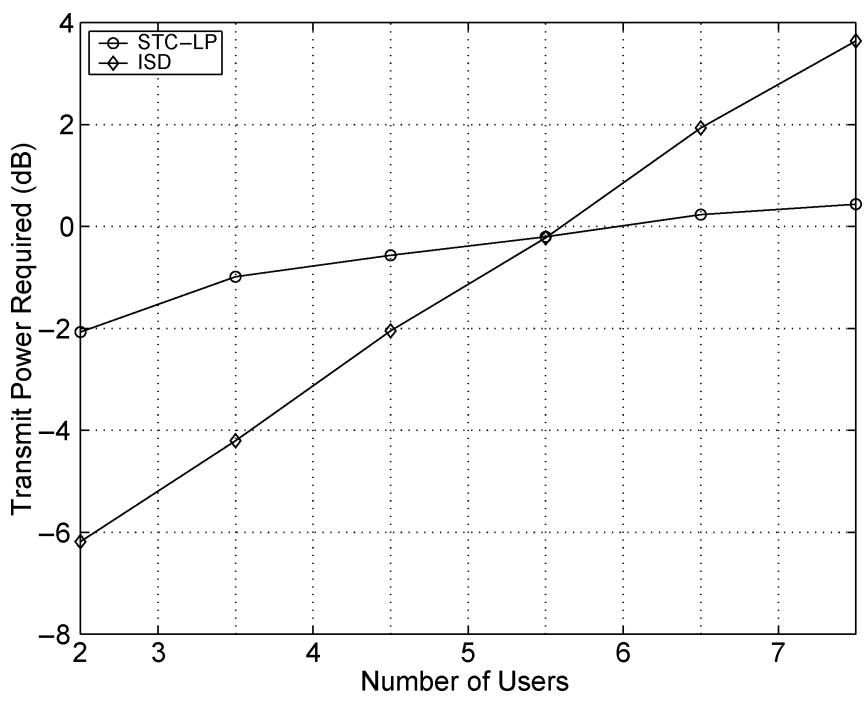

Fig. 9. Spatial optimization: $N=8$, flat fading channel. ISD algorithm is more likely to be trapped in a local minimum when the number of users is large.

$K=15$, the performance gain achieved by adding an antenna is $2.6 \mathrm{~dB}$ with ILDP algorithm and $2.1 \mathrm{~dB}$ with STC-LP algorithm. Here the tradeoff between the ILDP and STC-LP is better performance versus more efficient implementation. Although the STC-LP sustains about $0.5 \mathrm{~dB}$ loss in performance compared with the ILDP algorithm, it is much faster and simpler to implement.

\section{Space-Only Design}

In this section we examine the performance of the STC-LP and ISD algorithm for the spatial optimization problem. Fig. 9 shows the results for $N=8$ transmit antennas. When the number of users is small, ISD perform better than STC-LP. This is expected due to the suboptimal nature of the STC-LP algorithm. However, as the number of users goes up, the ISD algorithm performance degrades much faster than the STC-LP algorithm. This suggests that as the number of users increases, ISD algorithm is more likely to be trapped in a local minimum. On the other hand, the STC-LP algorithm performs quite well as the number of users increases. It only requires a moderate $2.3 \mathrm{~dB}$ of additional transmit power when the number of users increases from two to seven.

We next examine the performance gain obtained by adding more transmit antennas to the system. The STC-LP algorithm is used in this simulation. Fig. 10 shows when the number of transmit antennas goes from $N=4$ to $N=8$, there is a gain of approximately $2 \mathrm{~dB}$ for the entire range of the number of users considered. Equivalently, the power needed to satisfy five users when $N=4$ is enough to satisfy 17 users when $N=8$. Also note that another advantage of the STC-LP algorithm is that it can be used even when the number of users far exceeds the number of transmit antennas. For instance, in Fig. 10 the number of users $(K=20)$ is much larger than the number of antennas ( $N=4,6$, or 8$)$. The independence of the number of users makes the STC-LP algorithm more intuitively pleasing since one would expect that as the transmit power goes to infinity, we can satisfy an arbitrary number of users with arbitrary channel conditions and SNR requirements. 


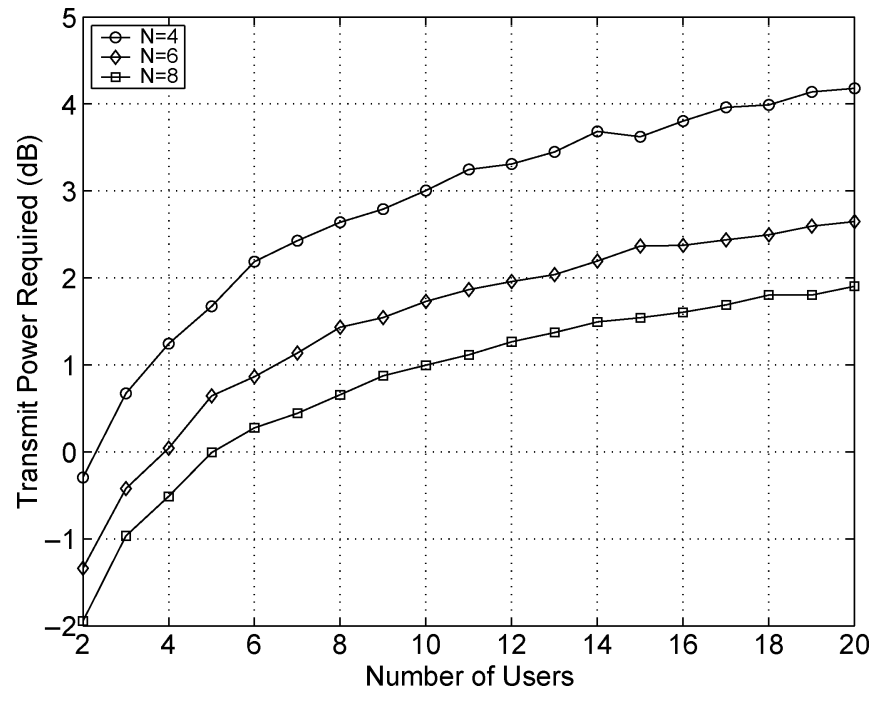

Fig. 10. Adding more antennas in spatial optimization. From $N=4$ to $N=$ 8 , the transmit power required to satisfy minimum received SNR drops by about $2 \mathrm{~dB}$.

\section{CONCLUSION}

The problem of optimal selective broadcast in a wireless CDMA system is studied in this work. It is shown that the optimal broadcasting scheme is more power efficient than the individual multiplexing transmission schemes and simple nonoptimal broadcasting schemes. We assume the channel state of all users are known at the transmitter, to facilitate the optimization of both the transmit signature code and receive filters. Space-time transmission methods for optimal broadcast are developed by investigating three different cases corresponding to time-only, space-time, and space-only design. The algorithms we developed include ILDP, LP/STC-LP, and ISD algorithms and offer tradeoffs between performance and complexity. We show that ILDP is applicable in both time-only and space-time design, whereas LP is initially developed for time-only design but can be extended to the STC-LP algorithm in space-time problem. We also develop an ISD algorithm that exploits the unique structure of the space-only design. The performance of various algorithms is evaluated by numerical simulation. The results show a $3.0 \mathrm{~dB}$ transmit power reduction with the ILDP or LP algorithm, compared to a simple nonoptimal broadcasting scheme. The transmit power can be further reduced by $2-2.5 \mathrm{~dB}$ via ILDP or STC-LP algorithm when the number of transmit antennas is increased from 1 to 2 .

\section{APPENDIX I \\ PROOF OF PROPOSITION 1}

We prove by contradiction. Suppose there is another set of vectors $\mathcal{C}^{o}=\left\{\mathbf{c}^{o}, \mathbf{r}_{1}^{o}, \ldots, \mathbf{r}_{K}^{o}\right\}$ that is a global minimizer for (5) and that performs better than $\mathcal{C}^{\text {opt }}=\left\{\mathbf{c}^{\text {opt }}, \mathbf{r}_{1}^{\text {opt }}, \ldots, \mathbf{r}_{K}^{\text {opt }}\right\}$ as defined in (6) and (7). This means $\mathbf{c}^{\mathrm{O}^{H}} \mathbf{c}^{\mathrm{O}}<\mathbf{c}^{\text {opt }}{ }^{H} \mathbf{c}^{\text {opt }}$, $\operatorname{Re}\left(\mathbf{r}_{k}^{o} \mathbf{H}_{k} \mathbf{c}^{o}\right) \geq \tilde{\eta}_{k}$, and $\left\|\mathbf{r}_{k}^{o}\right\|=1$ for $k=1, \ldots, K$. Now look at the set of vectors $\hat{\mathcal{C}}^{o}=\left\{\mathbf{c}^{o}, \tilde{\mathbf{r}}_{1}^{o}, \ldots, \tilde{\mathbf{r}}_{K}^{o}\right]$, where $\tilde{\mathbf{r}}_{k}^{o}=$ $\left(\mathbf{H}_{k} \mathbf{c}^{o} /\left\|\mathbf{H}_{k} \mathbf{c}^{o}\right\|\right)$. We claim that $\tilde{\mathcal{C}}^{o}$ performs the same as $\mathcal{C}^{o}$. We demonstrate this by noting that when going from $\mathcal{C}^{o}$ to $\tilde{\mathcal{C}}^{o}$ a) the cost function remains the same and b) none of the constraints is violated. It is apparent that condition a) holds by definition of $\tilde{\mathcal{C}}^{o}$. Meanwhile, it is easy to see that condition b) holds since $\tilde{\mathbf{r}}_{k}^{O^{H}} \mathbf{H}_{k} \mathbf{c}^{O}$ is nonnegative real by definition, and $\left|\tilde{\mathbf{r}}_{k}^{O^{H}} \mathbf{H}_{k} \mathbf{c}^{O}\right|^{2} \geq$ $\left|\mathbf{r}_{k}^{o^{H}} \mathbf{H}_{k} \mathbf{c}^{o}\right|^{2} \geq\left|\operatorname{Re}\left(\mathbf{r}_{k}^{o^{H}} \mathbf{H}_{k} \mathbf{c}^{o}\right)\right|^{2} \geq \tilde{\eta}_{k}^{2}$. The first inequality is a consequence of the Cauchy-Schwartz inequality. However, this also means $\mathbf{c}^{O}$ is just one of the feasible solutions for the problem stated in (6). Therefore, $\mathbf{c}^{\text {opt }}{ }^{H} \mathbf{c}^{\text {opt }} \leq \mathbf{c}^{\mathrm{O}^{H}} \mathbf{c}^{\mathrm{o}}$ since $\mathbf{c}^{\mathrm{opt}}$ is the minimum solution to (6). This contradicts the assumption and the proposition is proved.

\section{ACKNOWLEDGMENT}

The authors would like to thank Prof. John Gubner of the University of Wisconsin at Madison for helpful comments and suggestions.

\section{REFERENCES}

[1] J. Farber, "Network game traffic modeling," in Proc. 1st Workshop Netwrok and System Support for Games, 2002, pp. 53-57.

[2] H. Sari, G. Karam, and I. Jeanclaude, "Transmission techniques for digital terrestrial TV broadcasting," IEEE Commun. Mag., pp. 100-109, Feb. 1995.

[3] J. E. Wieselthier, G. D. Nguyen, and A. Ephremides, "Algorithms for energy-efficient multicasting in ad hoc wireless networks," in Proc. IEEE Military Communications Conf., vol. 2, 1999, pp. 1414-1418.

[4] A. J. Paulraj and B. C. Ng, "Space-time modems for wireless personal communications," IEEE Pers. Commun., pp. 36-48, Feb. 1998.

[5] J. W. Liang and A. J. Paulraj, "Forward link antenna diversity using feedback for indoor communications systems," in Proc. ICASSP-95, vol. 3, 1995, pp. 1753-1755.

[6] V. Tarokh, H. Jafarkhani, and A. R. Calderbank, "Space-time block codes from orthogonal designs," IEEE Trans. Inform. Theory, pp. 1456-1467, July 1999.

[7] S. M. Alamouti, "A simple transmit diversity technique for wireless communications," IEEE Trans. Select Areas Commun., pp. 1451-1458, Oct. 1998.

[8] D. P. Bertekas, Nonlinear Programming, 2nd ed. Nashua, NH: Athena Scientific, 1999

[9] C. L. Lawson and R. J. Hanson, Solving Least Squares Problems. Englewood Cliffs, NJ: Prentice-Hall, 1974.

[10] S. Lang, Undergraduate Analysis. Berlin, Germany: Springer-Verlag, 1997.

[11] T. M. Cover and J. A. Thomas, Elements of Information Theory. New York: Wiley Interscience, 1991.

[12] D. G. Luenberger, Optimization by Vector Space Methods. New York: Wiley, 1969.

[13] L. Scharf, Statistical Signal Processing: Detection, Estimation and Time Series Analysis. Reading, MA: Addison-Wesley, 1991.

[14] E. Onggosanusi, A. Gatherer, A. Dabak, and S. Hosur, "Performance analysis of close-loop transmit diversity in the presense of feedback delay," IEEE Trans. Commun., pp. 1618-1630, Sept. 2001.

[15] J. Tellado, Multicarrier Modulation With Low PAR, Applications to DSL and Wireless. Norwell, MA: Kluwer Academic, 2000.

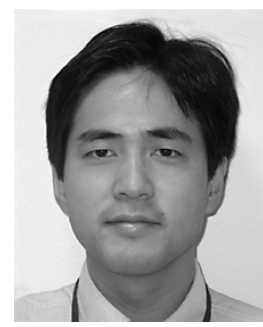

Jianzhong Zhang (S'96-M'04) received the B.S. degrees in electrical engineering and applied physics from Tsinghua University, Beijing, China, in 1995, the M.S. degree in electrical engineering from Clemson University, Clemson, SC, in 1998, and the Ph.D degree in electrical engineering from the University of Wisconsin at Madison in 2003.

His research has focused on the application of statistical signal-processing methods to wireless communication problems. Since 2001, he has been with Nokia Research Center, Irving, TX, where he worked on the transceiver designs for both EDGE and CDMA2000/WCDMA cellular systems. 


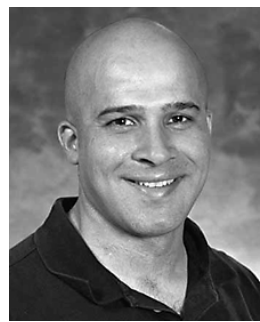

Akbar M. Sayeed (S'89-M'97-SM'02) received the B.S. degree from the University of Wisconsin at Madison in 1991 and the M.S. and Ph.D. degrees from the University of Illinois at Urbana-Champaign in 1993 an 1996, respectively, all in electrical and computer engineering.

While at the University of Illinois, he was a Research Assistant in the Coordinated Science Laboratory and was the Schlumberger Fellow in signal processing from 1992 to 1995. During 1996-1997, he was a Postdoctoral Fellow at Rice University, Houston, TX. Since August 1997, he has been with the University of Wisconsin at Madison, where he is currently Associate Professor of electrical and computer engineering. His research interests are in wireless communications, statistical signal processing, communication theory, information theory, and time-frequency analysis.

Dr. Sayeed received the NSF CAREER Award in 1999 and the ONR Young Investigator Award in 2001. He was an Associate Editor for the IEEE SIGNAL PROCESSING LETTERS from 1999 to 2002.

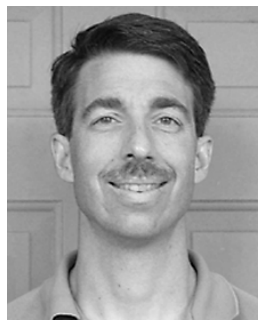

Barry D. Van Veen (S'81-M'86-SM'97-F'02) was born in Green Bay, WI. He received the B.S. degree from Michigan Technological University, Houghton, in 1983 and the Ph.D. degree from the University of Colorado, Boulder, in 1986, both in electrical engineering.

In the spring of 1987 he was with the Department of Electrical and Computer Engineering, University of Colorado. Since August 1987, he has been with the Department of Electrical and Computer Engineering, University of Wisconsin at Madison, where he currently holds the rank of Professor. His research interests include signal processing for sensor arrays, adaptive filtering, wireless communications, and biomedical applications of signal processing. He is coauthor (with S. Haykin) of Signals and Systems (New York: Wiley, 1999 and 2003).

Dr. Van Veen received a 1989 Presidential Young Investigator Award from the National Science Foundation and a 1990 IEEE Signal Processing Society Paper Award. He was an Associate Editor for the IEEE TRAnsactions ON Signal PROCESSING and a member of the IEEE Signal Processing Society's Technical Committee on Statistical Signal and Array Processing from 1991 through 1997. $\mathrm{He}$ is currently a member of the Sensor Array and Multichannel Technical Committee. He received the Holdridge Teaching Excellence Award from the Electrical and Computer Engineering Department at the University of Wisconsin in 1997. He was an ONR Fellow. 